\title{
Avaliando a experiência dos usuários com metodologias ativas nas aulas de Interação Humano-Computador
}

\author{
João Batista da Silva Junior ${ }^{1}$, Artur Kronbauer ${ }^{1,2}$, Jorge Campos ${ }^{1}$ \\ ${ }^{1}$ Programa de Pós-Graduação em Sistemas e Computação (PPGCOMP) - Universidade \\ Salvador (UNIFACS) - 41.770-235 - Salvador - BA - Brasil \\ ${ }^{2}$ Departamento de Ciências Exatas e da Terra (DCET II) - Universidade do Estado da \\ Bahia (UNEB) - 48.100-000 - Alagoinhas - BA - Brasil
}

joaojunior.mail@gmail.com, arturhk@gmail.com,jorgeapcampos@gmail.com

\begin{abstract}
This paper presents a study about students' experiences with active methodologies used during a Human-Computer Interaction course. The study was carried out with the participation of students of Computer Science and Information Systems during one semester. A computational platform composed of Web and mobile applications and a cloud database was used to collect and analyze the data from different self-report techniques. The information gathered with the pilot experiment allowed the identification of the hedonic feelings aroused in the students with the use of the different types of active methodologies. This kind of information is relevant to teachers to identify the pedagogical practices that most attract the participation of the students.
\end{abstract}

Resumo. Este artigo apresenta um estudo referente à experiência dos discentes em relação a um conjunto de metodologias ativas aplicadas nas aulas de um curso de Interação Humano-Computador. Para a coleta e análise dos dados foi utilizada uma plataforma que tem como infraestrutura um aplicativo para smartphones, uma base de dados em nuvem e disponibiliza diferentes técnicas de autorrelato para avaliar a experiência dos usuários. $O$ estudo foi realizado com a participação de alunos de Ciência da Computação e Sistemas de Informação no decorrer do semestre letivo. As informações obtidas com o experimento piloto possibilitaram identificar os sentimentos hedônicos despertados nos discentes com a utilização dos diferentes tipos de metodologias ativas, possibilitando ao professor identificar as práticas pedagógicas que mais atraem a participação dos alunos.

\section{Introdução}

Refletir sobre a educação no contexto contemporâneo perpassa, entre outros importantes aspectos, pela discussão sobre as Metodologias Ativas produzidas de forma colaborativa e as suas implicações nas experiências vivenciadas pelos discentes em sala de aula [Bacich e Moran, 2018]. As Metodologias Ativas possibilitam maior interação entre professores e alunos, beneficiando ambos. Os discentes adquirirem maior protagonismo e independência no processo de ensino-aprendizagem. Já os professores têm uma grande 
VIII Congresso Brasileiro de Informática na Educação (CBIE 2019)

Anais do XXX Simpósio Brasileiro de Informática na Educação (SBIE 2019)

oportunidade para inovar, propor novas formas de ensinar e estimular os seus alunos [Diesel et al., 2017].

$\mathrm{Na}$ literatura, é possível encontrar trabalhos que abordam as metodologias ativas como forma de potencializar o envolvimento e consequente aprendizado dos discentes, mas não identificamos trabalhos que identificam e analisam os sentimentos hedônicos provocados com a utilização destas metodologias.

Neste sentido, o objetivo desta pesquisa é descrever uma metodologia para analisar os sentimentos hedônicos provocados nos alunos ao serem submetidos a novas práticas pedagógicas. Como contribuições, espera-se que os passos executados na condução do experimento piloto sirvam de base para a realização de outras avaliações, conduzidas por professores de diferentes áreas, a fim de identificar os sentimentos hedônicos despertados em seus alunos durante a aplicação de novas práticas pedagógicas.

O restante deste artigo está estruturado da seguinte forma: na Seção 2, são discutidos alguns conceitos para posicionar o leitor em relação à proposta desta pesquisa, especialmente no que tange as técnicas de autorrelato para avaliação de User eXperience (UX) e as metodologias ativas utilizadas nas aulas de IHC; na Seção 3, é descrita a metodologia utilizada para a condução do experimento; a Seção 4 apresenta os resultados e discussões; e na Seção 5, são relatadas as conclusões e futuros trabalhos.

\section{Embasamento Teórico do Estudo de Caso}

As experiências hedônicas podem ser identificadas durante a realização de atividades comuns como, por exemplo, assistir a um filme, escutar música ou participar de uma aula. Estas atividades podem não propiciar uma boa experiência quando a música tem um ritmo desagradável, o enredo do filme é desinteressante ou a aula não propõem práticas pedagógicas envolventes. Nesse sentido, é importante identificar e entender as emoções geradas no público alvo, permitindo ajustá-las para tornar a experiência divertida, emocionante, interessante, agradável, satisfatória e memorável [Kaasinen, 2015].

Apesar do termo UX ter uma relação estreita com a área de Interação HomemComputador, seu conceito amplo permite analisar como as pessoas se sentem ao interagir com um produto ou serviço, independentemente de existir relação com dispositivos computacionais [Lachner et al., 2016]. Neste sentido, boas experiências por parte dos discentes com práticas pedagógicas adotadas pelos professores pode ser a chave para o estímulo e engajamento dos alunos no processo de ensino-aprendizagem.

Existem inúmeros métodos para a captura dos sentimentos hedônicos. Nesta pesquisa, entretanto, nos concentramos apenas nas técnicas de autorrelato, dado que fornecem métodos formalizados para que os próprios usuários informem seus sentimentos em relação a um produto ou serviço. Assim, a captura dos sentimentos hedônicos prescinde de algoritmos sofisticados para processamento automático de textos, imagens, gestos do usuário ou do uso de sensores invasivos para a medição da UX. Como desvantagem, as técnicas de autorrelato são menos oportunistas, isto é, elas necessitam da colaboração do usuário para a captura das emoções. 
VIII Congresso Brasileiro de Informática na Educação (CBIE 2019)

Anais do XXX Simpósio Brasileiro de Informática na Educação (SBIE 2019)

A restrição da utilização de técnicas de autorrelato na avaliação de UX ainda é muito abrangente, dado que estas técnicas são inúmeras e diversificadas. Desta forma, restringimos o escopo deste trabalho às técnicas de autorrelato disponibilizadas pela Plataforma UXSense, isto é: AtrackDiff [Hassenzahl, 2003] (Figura 1.A), Positive and Negative Affect Schedule (PANAS) [Watson, 1988] (Figura 1.B), Product Emotion Measure (PrEMO) [Desmet, 2005] (Figura 1.C) e Self-Assessment Manikin (SAM) [Bradley, 1994] (Figura 1.D). Existem na literatura diversas descrições de sucesso de avaliação de UX com a utilização destas técnicas de autorrelato. Em [Peedu e Lamas, 2011], por exemplo, foi utilizada a técnica AtrackDiff para capturar a UX dos usuários de sites governamentais após a reestruturação dos serviços. A técnica PANAS foi utilizada para provar a hipótese de que consumidores que estão em uma paixão romântica consomem uma variedade maior de produtos do que os que não estão [Huang, 2018]. A PrEMO, por sua vez, foi empregada para identificar a influência dos alimentos sobre as emoções hedônicas das pessoas [Dalenberg, 2014]. A técnica SAM foi usada em [Hayashi, 2016] para coletar as respostas afetivas de crianças relacionadas à qualidade dos sistemas educacionais.
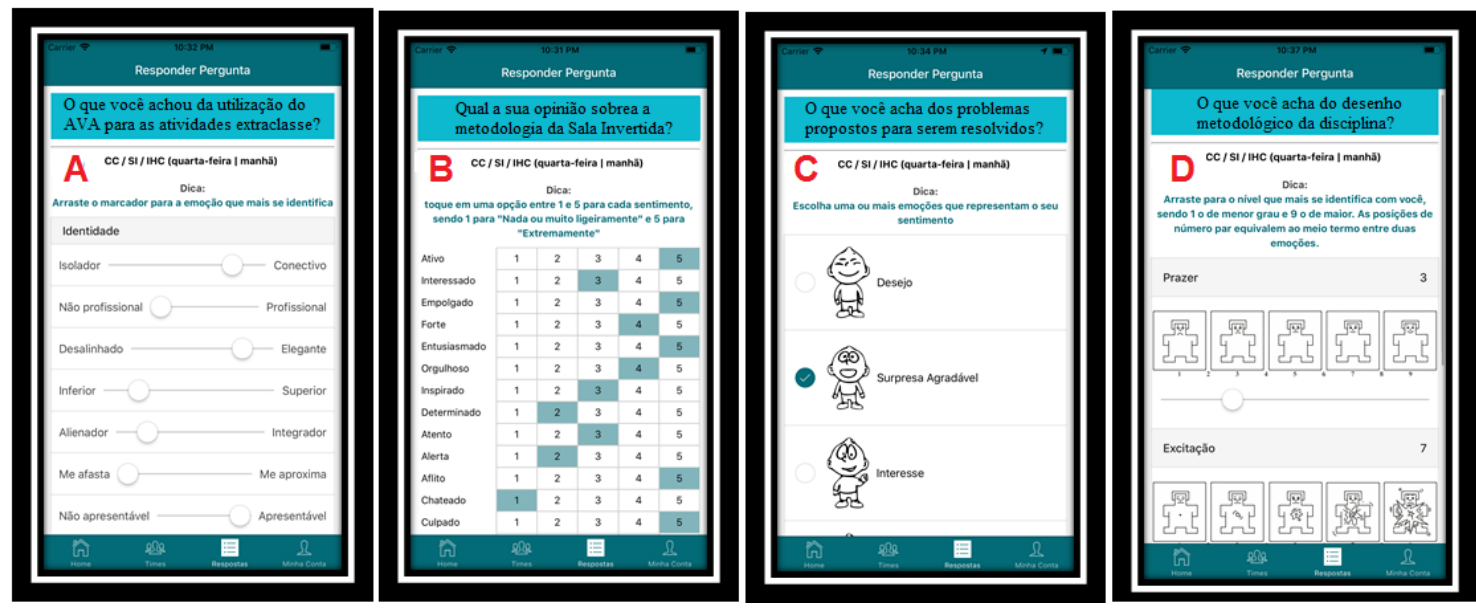

Figura 1. Técnicas de autorrelato utilizadas no contexto desta pesquisa.

No que diz respeito às metodologias ativas avaliadas no estudo de caso, o professor da disciplina de IHC propôs diferentes práticas pedagógicas com o objetivo de avaliar as experiências hedônicas despertadas nos discentes. Segundo a definição de [Diesel et al., 2017], as seguintes metodologias ativas de ensino-aprendizagem foram adotadas:

- Sala Invertida - Propõe a inversão completa do modelo de ensino. Esta metodologia é capaz de engajar os alunos no conteúdo ao ponto de os mesmos estarem aptos a lecionar. A metodologia Sala Invertida foi empregada na abordagem de assuntos específicos da disciplina de IHC. No método utilizado, os alunos deveriam ler previamente o conteúdo de um determinado tópico e alguns deles são selecionados para apresentar o tópico e conduzir a discussão em sala de aula.

- Aprendizagem Baseada em Problemas - Esta proposta defende a ideia de que a aprendizagem significativa deve ser baseada em apresentar problemas práticos para que os estudantes possam encontrar as soluções. Neste sentido, foram descritos cenários para os quais os discentes tinham que trazer soluções criativas de IHC que pudessem 
VIII Congresso Brasileiro de Informática na Educação (CBIE 2019)

Anais do XXX Simpósio Brasileiro de Informática na Educação (SBIE 2019)

contornar problemas de acessibilidade, multimodalidades de interações e projetos eficientes e eficazes.

- Aprendizagem Baseada em Projetos - Esta abordagem é utilizada para aprimorar a interdisciplinaridade e para enfatizar as atividades e habilidades dos participantes no desenvolvimento de artefatos. Os alunos foram solicitados a desenvolver mapas mentais na fase de projeto de interfaces de interação e prototipação de baixa fidelidade para esboçar a resolução dos problemas propostos na disciplina.

- Aprendizagem Baseada em Equipes - Esta metodologia propõe uma estratégia de ensino colaborativa aplicada em grupos. A ideia é que cada aluno tente entender individualmente os conceitos ensinados em sala de aula e reforce o aprendizado pela realização de atividades colaborativas com os demais colegas. Esta metodologia foi aplicada na elaboração de protótipos de alta fidelidade, contemplando produtos semiacabados para a resolução dos problemas propostos na disciplina.

- Atividade Extraclasse - Este mecanismo funciona como um complemento ao conteúdo ministrado em sala de aula e permite que os estudantes pratiquem e assimilem os temas abordados. A atividade foi desenvolvida em um Ambiente Virtual de Aprendizagem (AVA) utilizando a plataforma Blackboard. Foram disponibilizadas no AVA listas de discussões, vídeos, exercícios e chats online.

- Aprendizagem por Gamificação - Este conceito aplica a mecânica de competição para engajar e motivar os estudantes a participar das aulas. Esta metodologia foi empregada no final de conteúdos específicos abordados nas aulas, possibilitando que os alunos respondessem questionários utilizando uma aplicação específica para gamificação denominada de Kahoot. Os três alunos com maior pontuação nos testes foram bonificados com pontos extras na disciplina.

O estudo de caso para avaliação da experiência dos alunos com as metodologias ativas no curso de IHC será discutido na próxima seção.

\section{Metodologia aplicada para a realização do experimento piloto}

Para a elaboração do experimento piloto foram utilizados os procedimentos metodológicos indicados no framework DECIDE [Preece, 2005], cujas ações são: (i) determinar o objetivo da análise; (ii) explorar perguntas a serem respondidas; (iii) escolher o método de avaliação; (iv) identificar e administrar as questões práticas; (v) decidir como lidar com as questões éticas; e (iv) estabelecer forma de avaliar, interpretar e apresentar os resultados.

Com relação ao objetivo da análise, o foco do experimento piloto é avaliar os sentimentos hedônicos despertados nos discentes com a utilização das metodologias ativas proposta pelo professor da disciplina de IHC. O referido estudo contempla três turmas da mesma disciplina, totalizando 42 alunos.

Com respeito às perguntas a serem respondidas, foram especificadas cinco perguntas com o propósito de avaliar as experiências dos discentes quanto às metodologias utilizadas nas aulas (Tabela 1). As perguntas foram enviadas e exibidas aos participantes através de formulários eletrônicos da técnica de autorrelato 
VIII Congresso Brasileiro de Informática na Educação (CBIE 2019)

Anais do XXX Simpósio Brasileiro de Informática na Educação (SBIE 2019)

correspondente. As perguntas foram enviadas sistematicamente durante os horários das aulas.

Tabela 1. Perguntas elaboradas para o experimento.

\begin{tabular}{|l|l|l|}
\hline ID & \multicolumn{1}{|c|}{ Perguntas } & \multicolumn{1}{|c|}{ Objetivos } \\
\hline Q1 & $\begin{array}{l}\text { O que você acha de ministrar e promover a } \\
\text { discussão de conteúdos em sala de aula? }\end{array}$ & Sala Invertida \\
\hline Q2 & $\begin{array}{l}\text { O que você acha da criação de protótipos para } \\
\text { contemplar os assuntos abordados em aula? }\end{array}$ & $\begin{array}{l}\text { Aprendizagem Baseada em Problemas } \\
\text { Aprendizagem Baseada em Projetos } \\
\text { Aprendizagem Baseada em Equipes }\end{array}$ \\
\hline Q3 & O que você acha da utilização do AVA? & Atividade Extraclasse \\
\hline Q4 & $\begin{array}{l}\text { O que você acha da utilização de jogos } \\
\text { (Kahoot)? }\end{array}$ & Aprendizagem por Gamificação \\
\hline Q5 & $\begin{array}{l}\text { O que você acha do desenho metodológico da } \\
\text { disciplina? }\end{array}$ & Uso das Metodologias Ativas \\
\hline
\end{tabular}

$\mathrm{Na}$ identificação e administração das questões práticas, foram executados os seguintes passos: (i) selecionar as turmas que participariam do experimento piloto; (ii) apresentar as técnicas de captura de UX abordadas nesta pesquisa; (iii) preparar o cronograma de agendamento das perguntas e programar as técnicas de UX que seriam vinculadas a cada pergunta em função dos sentimentos que o professor da disciplina desejava identificar em cada metodologia; (iv) disponibilizar o aplicativo móvel para instalação; e (v) explicar e apresentar aos alunos informações sobre o experimento piloto e a plataforma.

Em virtude do experimento necessitar do envolvimento de seres humanos, a pesquisa foi encaminhada ao Comitê de Ética da Universidade, com aprovação pelo Parecer Consubstanciado de número 1.973 .433 e Certificado de Apresentação para Apreciação Ética (CAAE) de número 64728316.0.0000.5033.

Finalmente, para avaliar e interpretar os dados e apresentar os resultados foi utilizado o software Metabase, que é uma ferramenta de business intelligence (BI). Os resultados são apresentados em forma de gráficos e discutidos na próxima seção.

\section{Resultados e discussões do experimento piloto}

$\mathrm{Na}$ etapa de preparação do experimento, foi sugerido ao avaliador que elencasse os dois primeiros sentimentos antagônicos que lhe ocorriam quando mencionada cada uma das metodologias utilizadas nas aulas de IHC. Tomando como base as escolhas do docente, foram escolhidas as técnicas que apresentam maior potencial para identificar tais emoções [da Silva Júnior 2018]. Não se pretende defender aqui a utilização desta estratégia para preparação de avaliações da UX. Esta simplificação, no entanto, se justifica pelo fato de estarmos demostrando uma exemplificação de como realizar avaliações com técnicas de UX no contexto da sala de aula. Como esta área é multidisciplinar, a participação de psicólogos e pedagogos poderia ser apropriada para a escolha dos sentimentos e técnicas de autorrelato mais apropriadas para se definir as estratégias de avaliação. 
VIII Congresso Brasileiro de Informática na Educação (CBIE 2019)

Anais do XXX Simpósio Brasileiro de Informática na Educação (SBIE 2019)

\subsection{Análise da Sala Invertida}

Para avaliar a metodologia da Sala Invertida, foram sugeridos pelo professor os sentimentos de Entusiasmo e Vergonha. Desta forma, a equipe responsável pela avaliação escolheu a técnica PANAS que possui o maior potencial de avaliar tais sentimentos [da Silva Júnior 2018]. Nesta avaliação foram obtidas 52 respostas que foram qualificadas em uma escala de 5 níveis de intensidade, variando de "nada ou muito ligeiramente" até "extremamente".

A técnica PANAS captura 10 sentimentos positivos e 10 negativos (Figura 2). Em todos os gráficos deste artigo, os sentimentos positivos serão representados por círculos na cor verde e os negativos por quadrados na cor vermelha, facilitando a distinção de emoções antagônicas.

No que diz respeito aos dois sentimentos hedônicos escolhidos inicialmente pelo professor, foi possível constatar que o sentimento Entusiasmado teve uma média de 3.9 e Envergonhado 2.13, sendo possível verificar que o entusiasmo da maioria dos alunos superou a vergonha, o que é um ponto positivo para a aplicação desta metodologia.

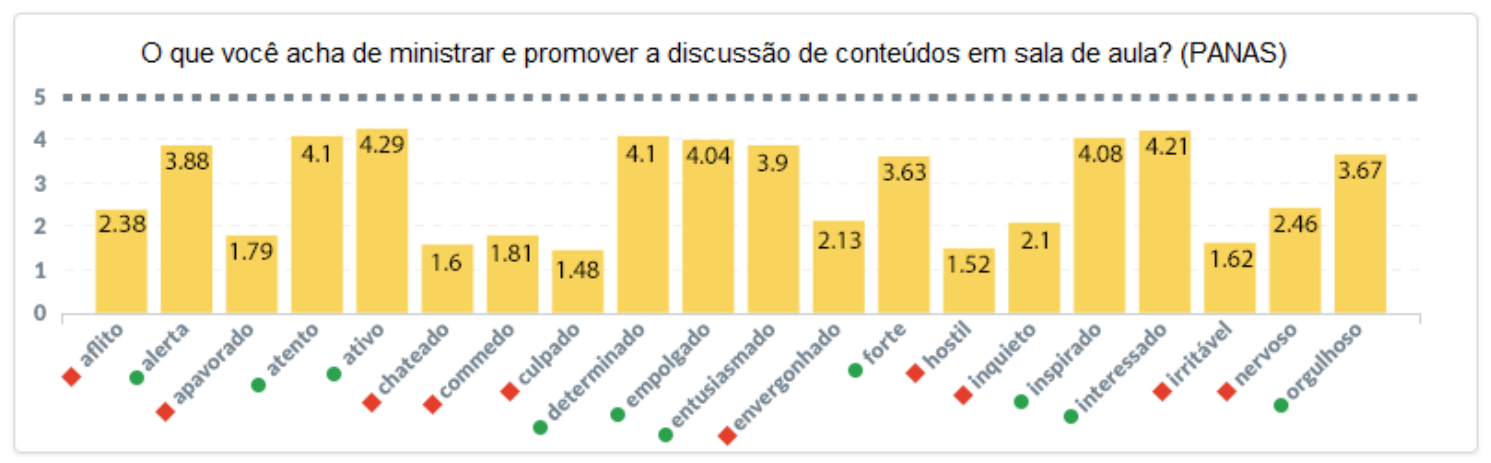

Figura 2. Resultado da avaliação da metodologia Sala Invertida.

De forma geral, é possível identificar que nenhum dos sentimentos positivos apresentou valores abaixo de 3 que é o valor da mediana na escala da técnica PANAS (valores de 1 a 5). Considerando os sentimentos negativos, todos apresentam valores abaixo do valor da mediana. Portanto, pode-se concluir que de forma geral a metodologia ativa da Sala Invertida proporcionou sentimentos hedônicos positivos para a maioria dos participantes do experimento.

\subsection{Análise da Aprendizagem Baseada em Problemas, Projetos e Equipes}

Os sentimentos sugeridos pelo professor para análise destas metodologias foram a Satisfação e a Frustração. Para este par de sentimentos, a equipe de avaliação sugeriu a técnica PrEMO.

Na técnica PrEMO, cada sentimento é representado com o tipo de dado booleano $($ verdadeiro $=1$ e falso $=0)$. Esta técnica possui 7 sentimentos positivos e 7 negativos o que permite ao usuário selecionar múltiplas emoções em uma única resposta (Figura 3). Assim, para obter os resultados foram somados o número de respostas para cada um dos sentimentos capturados pela técnica PrEMO (Figura 3).

$\mathrm{Na}$ análise dos resultados das avaliações das metodologias de análise da Aprendizagem Baseada em Problemas, Projetos e Equipes observa-se que a soma de 
VIII Congresso Brasileiro de Informática na Educação (CBIE 2019)

Anais do XXX Simpósio Brasileiro de Informática na Educação (SBIE 2019)

todos os sentimentos positivos totaliza 88 e dos negativos 9. Assim, conclui-se que a maioria dos estudantes tiveram sentimentos hedônicos positivos com as metodologias avaliadas.

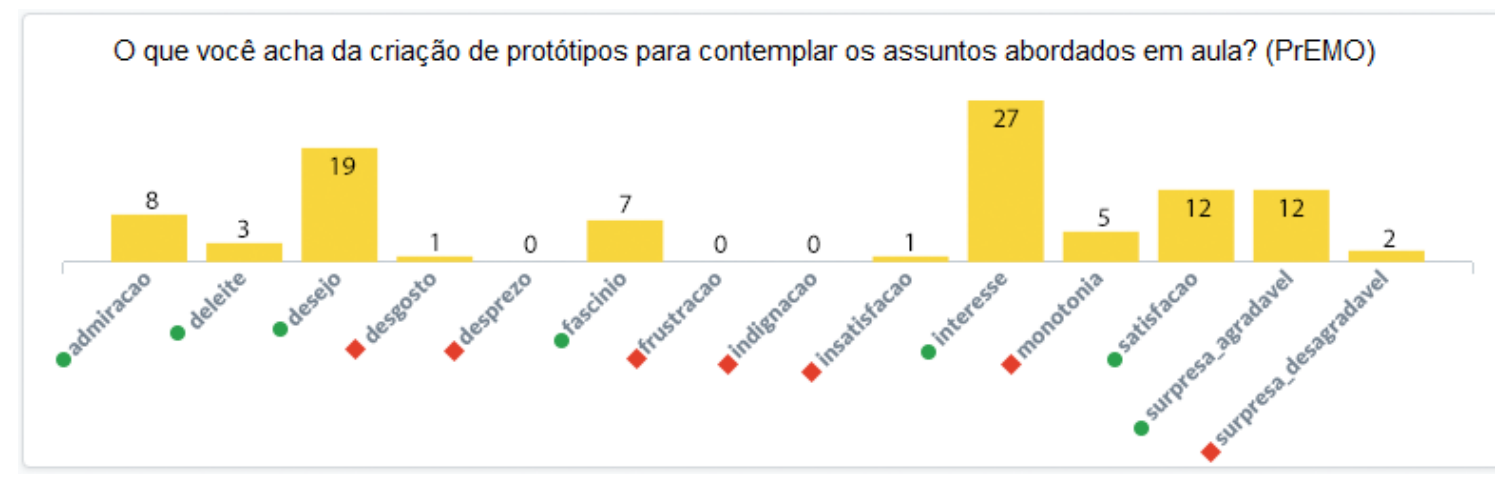

Figura 3. Resultado da avaliação das metodologias baseadas em Problemas, Projetos e Equipes.

Ao analisar os sentimentos sugeridos pelo professor, os resultados demonstram que ocorreram várias respostas indicando satisfação (12) em realizar os protótipos propostos na disciplina e nenhum dos alunos ficou frustrado (0), indicando boa aceitação das metodologias empregadas.

\subsection{Análise da Metodologia baseada em Atividade Extraclasse}

Para avaliar esta metodologia foram sugeridos pelo professor os sentimentos de Conectivo e Isolador, por se tratar de uma metodologia que se propõe a realizar atividades mediadas por tecnologia (AVA). Desta forma, a equipe de avalição indicou a técnica AttrakDiff em função da sua potencialidade em medir as referidas emoções.

A técnica AttrakDiff contempla 14 métricas positivas e 14 negativas. Conforme o gráfico da Figura 4 pode-se constatar que a maioria das métricas negativas possuem médias maiores quando comparadas às métricas positivas, com exceção das métricas Isolador-Conectivo que possuem a mesma média, e das métricas Alienador-Integrador e Profissional-Não Profissional cujas médias das métricas positivas superam as negativas.

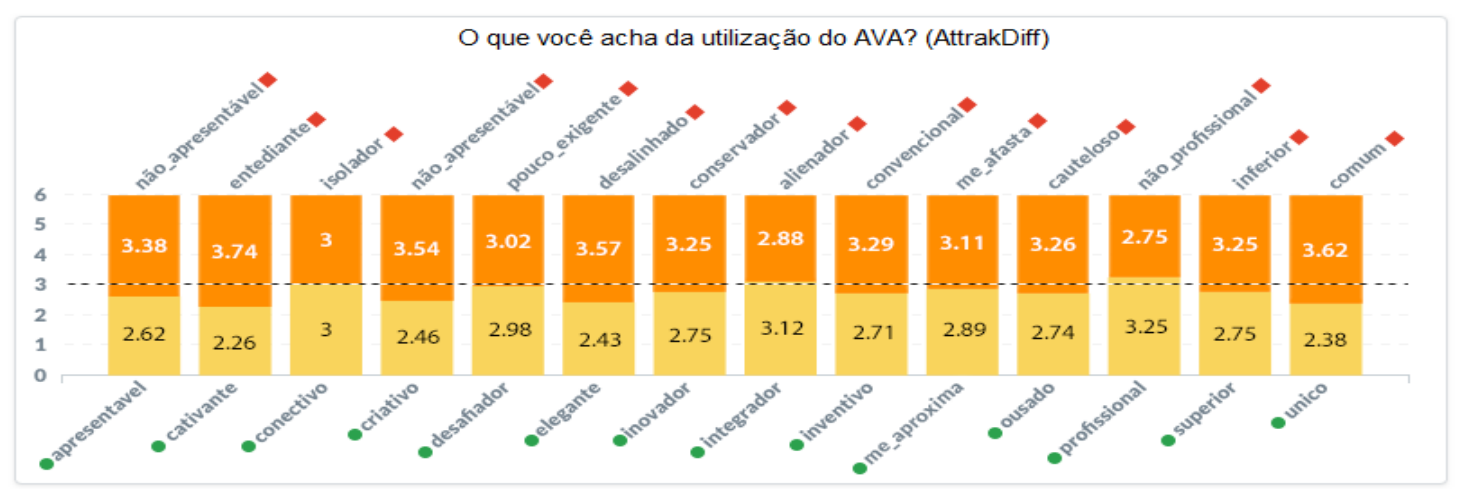

Figura 4. Resultado da avaliação da metodologia de Atividade Extraclasse.

De forma a sumarizar os valores para os sentimentos positivos e negativos, a média aritmética das métricas individuais foi utilizada novamente. $\mathrm{O}$ resultado foi 2,75 
VIII Congresso Brasileiro de Informática na Educação (CBIE 2019)

Anais do XXX Simpósio Brasileiro de Informática na Educação (SBIE 2019)

para as métricas positivas e 3,26 para as negativas, ou seja, a maioria dos alunos tiveram emoções hedônicas negativas em relação às atividades extraclasse. As atividades extraclasse consistiam-se basicamente de listas de discussões, vídeos, exercícios e chats online. Com relação aos sentimentos de Conectivo e Isolador, indicados pelo professor, não é possível chegar a uma constatação clara, dado que as métricas se mantiveram com médias iguais. Desta forma, não é possível identificar a influência da utilização do AVA como uma extensão da sala de aula.

\subsection{Análise da Metodologia de Aprendizagem por Gamificação}

Para a análise da metodologia por Gamificação, os sentimentos de Entusiasmo e Nervosismo foram escolhidos pelo professor, gerando mais uma vez a recomendação da técnica PANAS.

$\mathrm{Na}$ Figura 5, é possível observar que a maioria dos sentimentos positivos apresenta valores maiores do que os negativos. No entanto, para identificar a real diferença entre as emoções positivas e negativas, foi aplicada a média aritmética para consolidar todos os sentimentos de cada tipo de emoção (positivo e negativo). As emoções positivas deram o valor médio de 4,32 e as negativas 1,81. Desta forma, os dados indicam que as experiências hedônicas com a utilização de jogos em sala de aula geraram emoções positivas na maioria dos alunos, comprovando ser uma boa prática pedagógica.

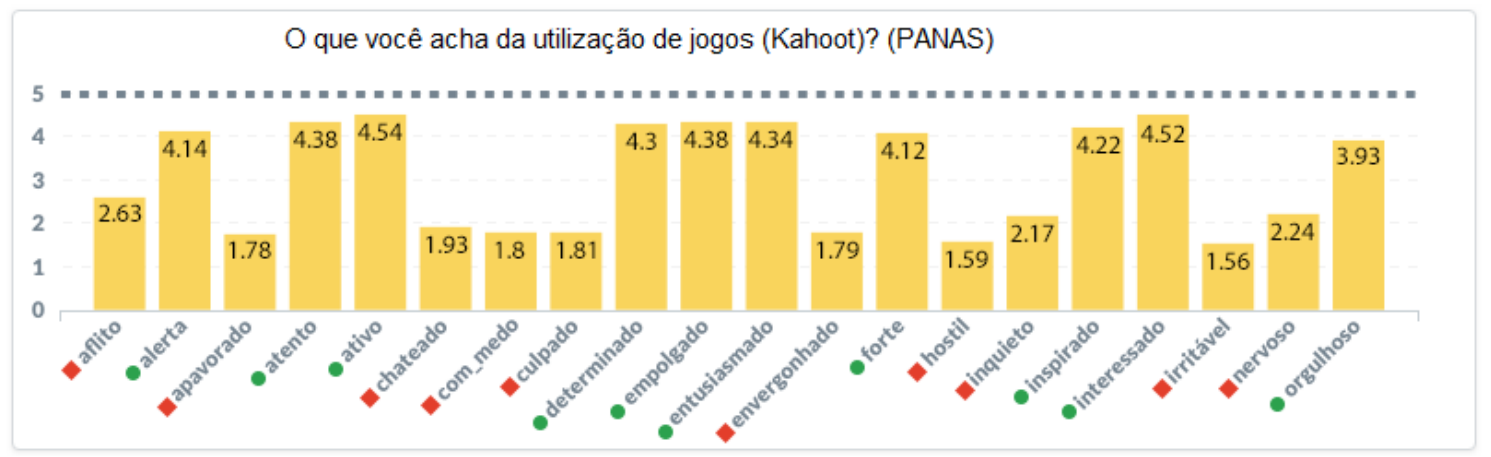

Figura 5. Resultado da avaliação da metodologia Baseada em Gamificação.

A análise dos dados do gráfico da Figura 5 possibilita afirma que a utilização de Gamificação no final da apresentação de cada tópico da disciplina gerou mais entusiasmo nos seus alunos do que nervosismo.

\subsection{Análise do geral da utilização das Metodologias Ativas}

Para avaliar a introdução de metodologias ativas nas aulas de IHC, a técnica SAM foi utilizada por disponibilizar três dimensões explicitas: prazer, excitação e dominância. A análise tomou como base a coleta de 101 respostas qualificadas na escala de 9 níveis de intensidade de acordo com a proposta da técnica. Para exibir os dados consolidados na escala da técnica SAM, foi aplicado a média aritmética sobre os valores capturados.

Os resultados apresentados na Figura 6 mostram que a maioria dos alunos obteve emoções hedônicas positivas acima da média nas três dimensões. Os dados mostram que $73,5 \%$ dos estudantes se mostraram com Dominância em relação às metodologias aplicadas, 69,5\% se sentiram Excitados e 79\% tiveram Prazer. Portanto, pode-se 
VIII Congresso Brasileiro de Informática na Educação (CBIE 2019)

Anais do XXX Simpósio Brasileiro de Informática na Educação (SBIE 2019)

concluir que a utilização das metodologias ativas proporcionou experiências agradáveis para a maioria dos estudantes.

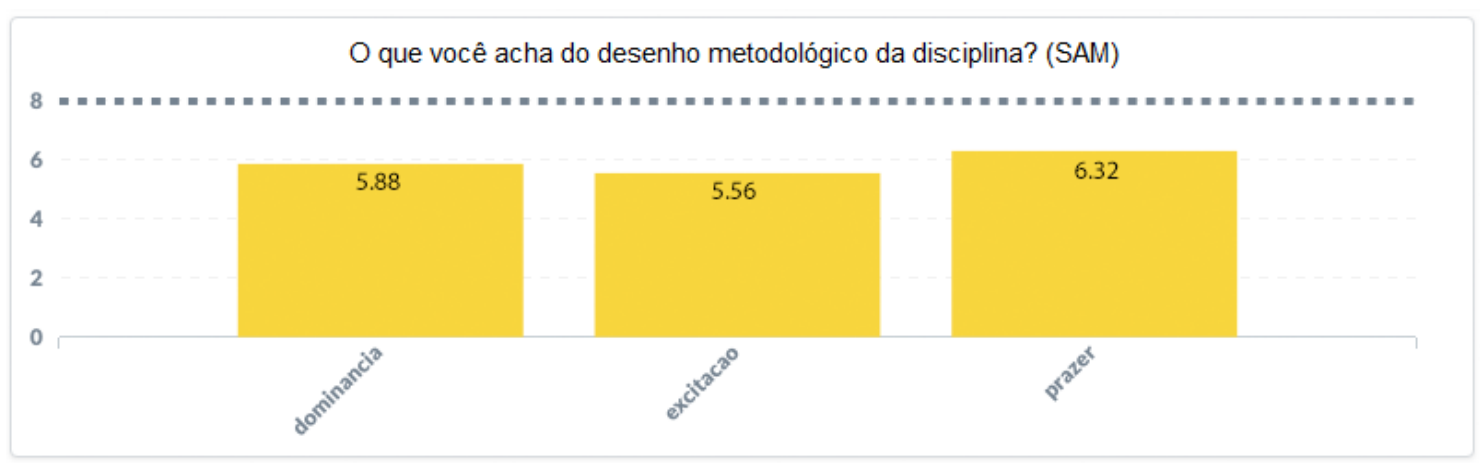

Figura 6. Resultado da utilização de metodologias ativas na disciplina de IHC.

\section{Conclusões e trabalhos futuros}

Diversas pesquisas estão sendo realizadas com o objetivo de analisar as potencialidades das metodologias ativas para o aprendizado ou facilidade de retenção do conhecimento por parte dos discentes. Entretanto, até o momento, existem poucos trabalhos que se proponham a avaliar a experiências dos alunos frente às metodologias ativas de ensino.

Neste artigo, foi apresentado um método para mensurar os sentimentos hedônicos envolvidos na aplicação de metodologias ativas como práticas pedagógicas, o que permite aos docentes identificar as metodologias que realmente despertam sentimentos positivos, favorecendo o engajamento dos discentes. A avaliação das metodologias ativas sob esta percepção proporciona ao professor criar os seus desenhos metodológicos com foco na satisfação do seu público alvo.

Nos resultados do experimento piloto, os alunos apresentaram sentimentos positivos para a maioria das metodologias ativas de ensino. Isto não significa que a simples adoção de metodologias ativas em sala de aula prescinde de um bom sincronismo de conteúdo e do apropriado desenho metodológico da disciplina. $\mathrm{O}$ entendimento da dinâmica em sala de aula e consequente adesão dos alunos estão profundamente relacionados ao sucesso da condução da disciplina. Além disso, os resultados do teste piloto não podem ser generalizados, porque o público alvo e o contexto foram escolhidos por conveniência. Esta particularidade, entretanto, não inviabiliza a contribuição deste trabalho que se propõe a descrever os passos para a replicação do experimento em diferentes contextos acadêmicos e permite aos docentes de diferentes áreas avaliarem as suas práticas pedagógicas sob a ótica da UX.

Como trabalhos futuros, pretende-se continuar as investigações em relação a adoção de metodologias ativas e os sentimentos hedônicos gerados aos discentes em disciplinas da área de computação, que apresentam grande impacto na evasão dos cursos, tais como, as disciplinas de cálculo e programação.

\section{Referências}

Bacich, L. and Moran, J. (2018). "Metodologias ativas para uma educação inovadora: uma abordagem teórico-prática”. Penso Editora. 
VIII Congresso Brasileiro de Informática na Educação (CBIE 2019)

Anais do XXX Simpósio Brasileiro de Informática na Educação (SBIE 2019)

Bradley, M. M. and Lang, P. J. (1994). "Measuring emotion: The selfassessment manikin and the semantic differential". Journal of Behavior Therapy and Experimental Psychiatry 25(1): 49-59.

Dalenberg, J. R., Gutjar, S., Horst, G. J., Graaf, K., Renken, R. J. and Jager, G. (2014). "Evoked emotions predict food choice". PloS one 9 (12), e115388.

da Silva Júnior, J. B. and Kronbauer, A. H. (2018). "A Study of Hedonic Experience Related to UX Capture Techniques". In Proceedings of the 17th Brazilian Symposium on Human Factors in Computing Systems, ACM, 4.

Desmet, P., Hekkert, P. and Jacobs, J. (2000). "When a car makes you smile: Development and application of an instrument to measure product emotions". Advances in consumer research 27 (1), 111-117.

Desmet, P. (2005). "Measuring emotions: Development and application of an instrument to measure emotional responses to products". In Funology: from usability to enjoyment. 111-123.

Diesel, A., Baldez, A. L. S. and Martins, S. N. (2017). “Os princípios das metodologias ativas de ensino: uma abordagem teórica". Revista Thema 14 (1), 268-288.

Hassenzahl, M., Burmester, M. and Koller, F. (2003). AttrakDiff: Ein Fragebogen zur Messung wahrgenommener hedonischer und pragmatischer Qualität. 187-196.

Hayashi, E., Posada, J. E. G, Maike, L and Baranauskas, M. C. (2016). Exploring new formats of the Self-Assessment Manikin in the design with children. In Proceedings of the 15th Brazilian Symposium on Human Factors in Computing Systems. ACM, 27.

Huang, X. and Dong, P. (2018). "Romantic Crushes Promote Variety-Seeking Behavior". Journal of Consumer Psychology, 29(2), 226-242.

Kaasinen, E., Roto, V., Hakulinen, J., Heimonen, T., Jokinen, P. P. J., Karvonen, Tuuli Keskinen, H., Koskinen, H., Lu, Y., Saariluoma, P., Tokkonen, H. and Turunen, M. (2015). "Defining user experience goals to guide the design of industrial systems". Behaviour \& Information Technology 34 (10): 976-991.

Lachner, F., Naegelein, P., Kowalski, R., Spann, M. and Butz A. (2016). "Quantified UX: Towards a Common Organizational Understanding of User Experience", proc. of the $9^{\text {th }}$ Nordic Conference on Human-Computer Interaction. ACM, 56.

Larson, R. and Csikszentmihalyi, M. (2014). "The experience sampling method". In Flow and the foundations of positive psychology. 21-34.

Peedu, G. and Lamas, D. (2011). Minu Viljandi: A case study on the effects of introducing web 2.0 features in e-government services on the overall user experience perception. In Proceedings of the 5th International Conference on Theory and Practice of Electronic Governance, ACM, 305-308.

Preece, J., Rogers, Y. and Sharp, H. (2005). "Design de interação". Bookman.

Russell, J. A., Weiss, A. and Mendelsohn G. A. (1989). "Affect Grid: A single-item scale of pleasure and arousal". Journal of Personality and Social Psychology 57 (3) 493-502.

Watson, D., Clark, L. A. and Tellegen, A. (1988). "Development and validation of brief measures of positive and negative affect: The PANAS scales". Journal of Personality and Social Psychology 54 (6), 1063-1070.

Xie, K., Heddy, B. C. and Greene, B. A. (2019). "Affordances of using mobile technology to support experience-sampling method in examining college students' engagement". Computers \& Education, Vol. 128, 183-198. 